\title{
Effect of leaf position and days post-infiltration on transient expression of colorectal cancer vaccine candidate proteins GA733-Fc and GA733-FcK in Nicotiana benthamiana plant
}

\author{
Kibum Kim ${ }^{1}$, Yang Joo Kang ${ }^{1}$, Se Ra Park ${ }^{1}$, Do-Sun Kim ${ }^{2}$, Seung-Won Lee ${ }^{1}$, Kinarm Ko ${ }^{3}$, Daniel Ponndorf ${ }^{4}$, \\ Kisung Ko ${ }^{\text {Corresp. } 1}$ \\ 1 Department of Medicine, Therapeutic Protein Engineering Lab, College of Medicine, Chung-Ang University, Seoul, South Korea \\ ${ }^{2}$ Vegetable Research Division, National Institute of Horticultural and Herbal Science, Rural Development Administration, Wanju-gun, South Korea \\ 3 Department of Stem Cell Biology, Konkuk University School of Medicine, Konkuk University, Seoul, South Korea \\ 4 Department of Biological Chemistry, John Innes Centre, Norwich Research Park, Colney, Colney, Norwich, UK \\ Corresponding Author: Kisung Ko \\ Email address: ksko@cau.ac.kr
}

Immunization with thetumor-associated antigen GA733 glycoprotein, which is highly expressed in colorectal cancer, is considered to be a promising strategy for cancer prevention and treatment. We cloned a fusion gene of GA733 and immunoglobulin Fc fragment (GA733-Fc), and that of GA733-Fc and an endoplasmic reticulum retention motif (GA733-FCK) into the Cowpea mosaic virus (CPMV)-based transient plant expression vector, pEAQ-HT. Agrobacterium tumefaciens (LBA4404) transformed with the vectors pEAQ-HT-GA733-Fc and pEAQ-HT-GA733-FcK was infiltrated into the leaves of Nicotiana benthamiana plants. To optimize harvesting of leaf to express therapeutic glycoproteins both spatially and temporally, protein expression levels at various leaf positions (top, middle, and base) and days post-infiltration (dpi) were investigated. The GA733-Fc and GA733-FcK genes were detected in leaves at 1-10 dpi using PCR. As assessed by western blot, GA733-Fc and GA733-FcK were expressed at the highest levels in the top leaf position at $5 \mathrm{dpi}$, and GA733-FcK was expressed more than GA733-Fc. The proteins were successfully purified from infiltrated $N$. benthamiana leaves using protein $A$ affinity chromatography. ELISA verified that an anti-GA733 antibody recognized both purified proteins. Thus, a functional GA733-Fc colorectal cancer vaccine protein can be transiently expressed using a CPMV virus-based vector, with an optimized expression time and leaf position post-infiltration. 


\title{
Effect of leaf position and days post-infiltration on transient expression of colorectal cancer vaccine candidate proteins GA733-Fc and GA733-FcK in Nicotiana benthamiana plant
}

\author{
Kibum Kim ${ }^{1}$, Yang Joo Kang ${ }^{1}$, Se Ra Park ${ }^{1}$, Do-Sun Kim² ${ }^{2}$, Seung-Won Lee ${ }^{1}$, Kinarm Ko ${ }^{3}$, \\ Daniel Ponndorf ${ }^{4}$, and Kisung Ko* \\ 1 Department of Medicine, Therapeutic Protein Engineering Lab, College of Medicine, \\ Chung-Ang University, 84 Heukseok-ro Dongjak-gu, Seoul 156-756, Korea \\ 2 Vegetable Research Division, National Institute of Horticultural and Herbal Science, \\ Rural Development Administration, Wanju-gun, South Korea \\ 3 Department of Stem Cell Biology, Konkuk University School of Medicine, Seoul, \\ Korea \\ 4 Department of Biological Chemistry, John Innes Centre, Norwich Research Park, Colney, \\ Norwich, UK \\ * Corresponding Author: \\ Kisung Ko \\ Department of Medicine, Therapeutic Protein Engineering Lab, College of Medicine, \\ Chung-Ang University, 84 Heukseok-ro Dongjak-gu, Seoul 156-756, Korea \\ Email address: ksko@,cau.ac.kr
}

\section{Abstract}

Immunization with the tumor-associated antigen GA733 glycoprotein, which is highly expressed in colorectal cancer, is considered to be a promising strategy for cancer prevention and treatment. We cloned a fusion gene of GA733 and immunoglobulin Fc fragment (GA733-Fc), and that of GA733-Fc and an endoplasmic reticulum retention motif (GA733-FcK) into the Cowpea mosaic virus (CPMV)-based transient plant expression vector, $\mathrm{pEAQ}-H T$. Agrobacterium tumefaciens (LBA4404) transformed with the vectors pEAQ- $H T$-GA733-Fc and pEAQ- $H T$-GA733-FcK was infiltrated into the leaves of Nicotiana benthamiana plants. To optimize harvesting of leaf to express therapeutic glycoproteins both spatially and temporally, protein expression levels at various leaf positions (top, middle, and base) and days post-infiltration (dpi) were investigated. The GA733-Fc and GA733-FcK genes were detected in leaves at 1-10 dpi using PCR. As assessed by western blot, GA733-Fc and GA733-FcK were expressed at the highest levels in the top leaf position at $5 \mathrm{dpi}$, and $\mathrm{GA} 733-\mathrm{FcK}$ was expressed more than GA733-Fc. The proteins [GA733-Fc (1.12 mg/g of leaf) and GA733-FcK (11.82 mg/g of leaf)] were successfully purified from infiltrated $N$. benthamiana leaves using protein A affinity chromatography. ELISA verified that an anti-GA733 antibody recognized both purified proteins. Thus, a functional GA733-Fc colorectal cancer vaccine protein can be transiently expressed using a CPMV virus-based vector, with an optimized expression time and leaf position post-infiltration.

Keywords: Agroinfiltration, fusion protein, KDEL, molecular biopharming, recombinant vaccine 
42

43

44

45

46

47

48

49

50

51

52

53

54

55

56

57

58

59

60

61

62

63

64

65

66

67

68

69

70

71

72

73

74

75

76

77

78

79

80

81

82

83

84

85

\section{Introduction}

Tumor-associated antigens (TAAs) are promising immunotherapeutic vaccine candidate proteins for cancer prevention and treatment ( $\mathrm{Lu}$ et al. 2012; Wong-Arce et al. 2017). Immune responses for induction of immunoglobulins to target TAAs have been actively studied (Eun-Wha Son et al. 2005). Among TAAs, glycoprotein GA733, or epithelial cell adhesion molecule (EpCAM), is highly expressed on the surface of colorectal cancer cells and has been extensively investigated (Eyvazi et al. 2018; Lu et al. 2012). Anti-GA733 antibody has been shown to inhibit the growth of colorectal cancer cells, which results from GA733 immunization in mice (Brodzik et al. 2008). The extracellular domain of GA733 is often used as a target for colorectal cancer vaccination and is fused with a human immunoglobulin Fc fragment (GA733-Fc) to enhance immune responses (Kim et al. 2018; Lee et al. 2017). The presence of the Fc domain markedly increases the plasma half-life of the protein, which prolongs therapeutic activity (Czajkowsky et al. 2012), and it simplifies purification of the protein by binding to protein A (Lee et al. 2020; Park et al. 2020).

Plants have several advantages over existing mammalian cell-based systems in terms of lower production costs and the absence of human pathogen contamination (Chen et al. 2013; Lee et al. 2017; Loh \& Wayah 2014; Shin et al. 2019). In addition, plants require a simple cultivation environment (sunlight, water, nutrients, and fertilizer) to grow (Lim et al. 2015; Rigano et al. 2005). Moreover, unlike bacterial cells, plants have post-translational modification systems similar to those of mammalian cells, such as glycosylation. Since plant cells are eukaryotic and have an endomembrane system and secretory pathway (Loh \& Wayah 2014), plants can produce and assemble correctly folded and complex mammalian proteins in their cells (Menassa et al. 2012; Se-Ra Park 2019). Thus, plants are considered as an attractive alternative for the production of therapeutic recombinant proteins (Lee et al. 2017; Nausch et al. 2012). In our previous study, plants have been determined to highly produce recombinant GA733-Fc fusion proteins (Park et al. 2015).

In general, there are two approaches to express recombinant proteins in plants, which are transgenic and transient plant expression (Chen et al. 2013; Joh et al. 2006; Ma et al. 2019; Wydro et al. 2006). For transient expression, the vector pEAQ-HT, which is based on the Cowpea mosaic virus (CPMV), has been reported to be an efficient expression vector for therapeutic recombinant proteins (Shah et al. 2013). This deconstructed vector lacks the viral coat protein and has been shown to allow high and fast recombinant protein production (Chen et al. 2013; Daniell et al. 2009; Peyret \& Lomonossoff 2013). In addition, Agrobacterium-mediated transient expression using a syringe or vacuum machine to transfer the CPMV-based pEAQ vector can be faster, cheaper, and more convenient than traditional stable transformation systems, without the need for viral replication (Loh \& Wayah 2014). However, the expression period and plant tissue position can influence both the quality and quantity of the recombinant protein (Lim et al. 2015; Yamamoto et al. 2018).

Thus, the goal of our current research is to establish spatial and temporal conditions for the maximum production of the colorectal cancer vaccine candidate proteins GA733-Fc and GA733Fc with an ER retention motif (GA733-FcK), using the CPMV-based pEAQ transient expression system. In order to study optimal expression of both GA733-Fc and GA733-FcK, the GA733-Fc and GA733-FcK expression vectors were transferred to plant leaves in a laboratory scale using a direct-syringe agroinfiltration method. Gene insertion, protein expression, protein purification, and antibody recognition of both recombinant proteins were investigated along with day postinfiltration and leaf position. 
86

87

88

89

90

91

92

93

94

95

96

97

98

99

100

101

102

103

104

105

106

107

108

109

110

111

112

113

114

115

116

117

118

119

120

121

122

123

124

125

\section{Materials \& Methods}

Plasmid construction

To construct the pEAQ vectors for transient expression of GA733, the sequence encoding the GA733 was ligated to that of human IgG Fc to generate the GA733-Fc fusion gene, and this recombinant gene was tagged with an ER retention signal (KDEL) to generate GA733-FcK (Fig. 1). These sequences were cloned individually into the pEAQ- $H T$ vector (Peyret \& Lomonossoff 2013; Sainsbury et al. 2009), which is based on CPMV, a non-enveloped plant virus in the Comovirus family consisting of RNA-1 $(6.0 \mathrm{~kb})$ and RNA-2 $(3.5 \mathrm{~kb})$ molecules (Peyret \& Lomonossoff 2013). The T-DNA region of the pEAQ- $H T$ vector possesses the suppressor of gene silencing, P19, and the gene encoding neomycin phosphotransferase III for resistance to kanamycin (Sainsbury et al. 2009). The GA733-Fc and GA733-FcK sequences were cloned into the AgeI site of pEAQ- $H T$ using the In-Fusion ${ }^{\circledR}$ HD Cloning Kit (Takara, Mountain View, CA) (Fig. 1). In the recombinant $\mathrm{pEAQ}-H T$ vectors, expression of GA733-Fc and GA733-FcK was under the control of the Cauliflower mosaic virus $35 \mathrm{~S}$ promoter and nopaline synthase terminator (Sainsbury et al. 2009) (Fig. 1). Agrobacterium tumefaciens (LBA4404) was transformed with the recombinant binary plasmid vectors, and the resulting transformants were named Ag/pEAQGA733-Fc and Ag/pEAQ-GA733-FcK.

Plants for agroinfiltration

$N$. benthamiana plant was used for agroinfiltration. The seeds of $N$. benthamiana were sown in commercial, nitrogen-fertilized soil (Seoul Bio, Chungcheongbuk-do, Korea). After 14 days, the seedlings were transplanted into pots containing nitrogen-fertilized soil. They were grown in a plant chamber at $25^{\circ} \mathrm{C}$ with $16 \mathrm{~h}$ of light and $8 \mathrm{~h}$ of darkness. Water was supplied every 2 days $(50-100 \mathrm{ml})$. The plants were used for experiments after 6 weeks.

Agroinfiltration using the syringe method

Agrobacteria carrying each vector pEAQ-GA733-Fc and pEAQ-GA733-FcK were subcultured on LB agar containing antibiotics $\left(10 \mu \mathrm{g} / \mathrm{ml}\right.$ kanamycin) at $28^{\circ} \mathrm{C}$ for $48 \mathrm{~h}$. Then, 100 $\mu \mathrm{l}$ of infiltrating medium was inoculated in $40 \mathrm{ml}$ of fresh LB medium with $10 \mu \mathrm{g} / \mathrm{ml} \mathrm{kanamycin}$ and incubated for $21-23 \mathrm{~h}$ at $28^{\circ} \mathrm{C}$ with shaking at $210 \mathrm{rpm}$ until 0.35 optical density at $600 \mathrm{~nm}$ was obtained. The agrobacteria were pelleted by centrifugation at $3,000 \mathrm{rpm}$ followed by resuspension in $40 \mathrm{ml}$ of MMA buffer (10 mM 2-N-morpholinoethanesulfonic acid, $\mathrm{pH}$ 5.6, 10 $\mathrm{mM} \mathrm{MgCl}, 100 \mu \mathrm{M}$ acetosyringone) (Sainsbury et al. 2009). The abaxial side of each $N$. benthamiana leaf was pricked using a syringe needle to aid infiltration. Then, MMA buffer containing Ag/pEAQ-GA733-Fc or Ag/pEAQ-GA733-FcK was infiltrated into the undersides of each leaf with soft pressure using a $1 \mathrm{ml}$ syringe (needle removed) until the entire leaf was wet (Loh \& Wayah 2014). The plant leaves with the same age were used for agrobacterium inoculation. The same leaf size was used for each location grown during the same period. After infiltration, the plants were grown at $25^{\circ} \mathrm{C}$ in a chamber and watered every 1-2 days to prevent drying. The infiltrated leaves were harvested as described directly below. All samples were taken in triplicate with the same leaf at the same location for each dpi.

Harvesting agroinfiltrated leaves in accordance with days post-infiltration (dpi)

Peer) reviewing PDF | (2020:08:51767:1:1:NEW 22 Dec 2020) 
$N$. benthamiana leaf samples were collected at $1,3,5,7,8,9$, and 10 dpi to determine timedependent recombinant protein expression levels. The infiltrated top, middle, and base leaves were harvested to investigate spatial protein expression and morphological changes.

Protein extraction and western blot analysis

The agroinfected leaves were harvested according to leaf positions (top, middle, and base leaves) for western blot analysis. After harvesting, the leaf blades were washed with tap water and gently dried with tissue paper. The harvested leaves $(100 \mathrm{mg})$ were homogenized in three volumes (w/v) of $1 \times$ PBS $\left(2.7 \mathrm{mM} \mathrm{KCl}, 137 \mathrm{mM} \mathrm{NaCl}, 10 \mathrm{mM} \mathrm{Na}_{2} \mathrm{HPO}_{4}\right.$ and $\left.2 \mathrm{mM} \mathrm{KH}_{2} \mathrm{PO}_{4}\right)$ to extract the TSP. Two microliters of each sample was mixed with $0.4 \mu \mathrm{l}$ of loading buffer $(1 \mathrm{M}$ Tris- $\mathrm{HCl}$, $10 \%$ SDS, $5 \% 2$-mercaptoethanol, $50 \%$ glycerol, and $0.1 \%$ bromophenol blue) and $16.6 \mu \mathrm{l}$ of distilled water. The mixed samples were subjected to $10 \%$ SDS-PAGE in SDS running buffer $(0.2$ $\mathrm{M}$ glycine, $25 \mathrm{mM}$ Tris- $\mathrm{HCl}, 0.1 \%$ [w/v] SDS). The protein gel was transferred to a nitrocellulose membrane (Millipore Corp, Billerica, MA). The blotted membranes were transferred to $1 \times$ PBST buffer ( $1 \times$ PBS with $0.5 \%$ [v/v] Tween 20 ) and then blocked in 5\% skim milk (Sigma, St. Louis, $\mathrm{MO})$ for $2 \mathrm{~h}$ at room temperature. The membrane was incubated with primary mouse anti-EpCAM $\mathrm{mAb}$ (anti-GA733 $\mathrm{mAb}$ ) (R\&D Systems, MN) (Isotype $\operatorname{IgG}_{2 \mathrm{a}}, 1: 5,000$ dilution) to detect the GA733 portion of GA733-Fc and GA733-FcK, and then with 1:5,000-diluted secondary HRPconjugated anti-mouse $\operatorname{IgG}_{2 \mathrm{a}}$ (Abcam, Cambridge, UK), each for $2 \mathrm{~h}$ at room temperature with shaking $(110 \mathrm{rpm})$. The protein bands were detected by using SuperSignal Chemiluminescent Substrate (Pierce, Rockford, IL) and visualized by exposure to X-ray film (Fuji, Tokyo, Japan). EpCAM-Fc from mammalian cell $\left(\mathrm{GA} 733-\mathrm{Fc}^{\mathrm{M}}\right)$ (rhEpCAM/Fc Chimera, R\&D Systems, MN) was used as a positive control, and non-transformed $N$. benthamiana leaves were used as a negative control (Lim et al. 2015).

\section{Genomic DNA isolation and PCR}

Genomic DNA in transfected leaves was isolated using the Genomic DNA Mini Kit (Favorgen, Taiwan), according to the manufacturer's instructions. PCR was conducted to confirm the presence of the GA733-Fc and GA733-FcK genes in the agroinfected leaves using a PCR premix kit (iNtRON Biotechnology, Seoul, Korea). The sequences of the primers were as follows: GA733-Fc forward primer, 5'-CAA ATT CGC GAC CGG ATG GCT ACT CAA CGA AGG-3' and reverse primer, 5'-GGT GAT GCA TAC CGG TCA ACC CGG GGA CAG GGA-3'; GA733FcK forward primer, 5'-CAA ATT CGC GAC CGG ATG GCT ACT CAA CGA AGG-3' and reverse primer, 5'-GGT GAT GCA TAC CGG TCA GAG TTC ATC TTT ACC C-3'. The PCR consisted of an initial denaturation at $94^{\circ} \mathrm{C}$ for $20 \mathrm{~min}$, followed by 30 cycles of denaturation at $94^{\circ} \mathrm{C}$ for $20 \mathrm{~s}$, annealing at $64^{\circ} \mathrm{C}$ for $30 \mathrm{~s}$, and extension at $72^{\circ} \mathrm{C}$ for $2 \mathrm{~min}$, and then a final extension at $72^{\circ} \mathrm{C}$ for $2 \mathrm{~min}$. Non-transfected plants were used as a negative control, while vectors carrying the expression cassettes of GA733-Fc and GA733-FcK in Escherichia coli were used as positive controls.

\section{Purification of GA733-IgG Fc and GA733-IgG FcK proteins from agroinfected leaves}

To purify GA733-Fc and GA733-FcK, $100 \mathrm{~g}$ of agroinfected leaves were homogenized in extraction buffer (37.5 mM Tris-HCl, pH 7.5, $50 \mathrm{mM} \mathrm{NaCl}, 15 \mathrm{mM}$ EDTA pH 8.0, $75 \mathrm{mM}$ sodium citrate, and $0.2 \%$ sodium thiosulfate) using an aluminum blender (Hanil, Seoul, Korea) and then centrifuged at $7,000 \times g$ for $30 \mathrm{~min}$ at $4^{\circ} \mathrm{C}$. The supernatant was collected, further clarified using Miracloth (Biosciences, La Jolla, CA), and adjusted to $\mathrm{pH} 5.1$ with acetic acid. The protein solution 
169 was then centrifuged at $10,000 \times g$ for $30 \mathrm{~min}$. The supernatant was collected, further clarified 170 using Miracloth, and adjusted to $\mathrm{pH} 7.0$ with $3 \mathrm{M}$ Tris-HCl. Eight percent of the total supernatant 171 volume of ammonium sulfate was added to the supernatant, and the solution was incubated at $4^{\circ} \mathrm{C}$ 172 for $2 \mathrm{~h}$, and then $22.6 \%$ ammonium sulfate was added to the total solution volume. The mixture 173 was incubated overnight at $4^{\circ} \mathrm{C}$ and then centrifuged at $8,000 \times g$ for $30 \mathrm{~min}$ at $4^{\circ} \mathrm{C}$. The pellet 174 was resuspended in $1 / 10$ of the original volume of extraction buffer, and then centrifuged again at $1758000 \times g$ for $30 \mathrm{~min}$ at $4^{\circ} \mathrm{C}$. The supernatant was filtered through a $0.45-\mu \mathrm{m}$ syringe filter 176 (MILLEX, Germany) and loaded on a protein A resin column (Sepharose 4 Fast Flow, GE 177 Healthcare, Sweden, NJ). The target proteins were eluted using elution buffer according to the manufacturer's recommendations. The final eluted protein fractions were subjected to SDS-PAGE to assess their purity. The gel was stained with Coomassie blue staining solution $[10 \%$ acetic acid $(\mathrm{v} / \mathrm{v}), 30 \%$ methanol (v/v), and $0.01 \%$ Coomassie blue (w/v)] at room temperature for 30 min with shaking. The gel was destained with destaining solution (10\% acetic acid and $30 \%$ methanol) thrice for $30 \mathrm{~min}$ each with shaking. The stock GA733-Fc and GA733-FcK protein solutions were dialyzed three times against sterile $1 \times$ PBS.

ELISA to confirm the ability of GA733-Fc and GA733-FcK to bind anti-GA733 antibody

MaxiSorp ${ }^{\text {TM }}$ 96-well ELISA plates (Nunc, Rochester, NY) were coated with $100 \mu 1$ per well of carbonate-bicarbonate (Sigma-Aldrich, St. Louis, MO) buffer containing mouse anti-EpCAM $\mathrm{mAb}$ (anti-GA733 mAb) (R\&D Systems, MN) (50 ng) and were incubated overnight at $4^{\circ} \mathrm{C}$. The plates were washed four times with $1 \times$ PBS-T [ $1 \times$ PBS plus $0.5 \%(v / v)$ Tween 20$]$. After washing, serially diluted GA733-Fc and GA733-FcK protein samples (12.5 ng to $0.196 \mathrm{ng}$ ) were added to each well, and then the plates were incubated for $2 \mathrm{~h}$ at $37^{\circ} \mathrm{C}$. The wells were washed four times with $1 \times$ PBS-T. One hundred microliters of HRP-conjugated goat anti-human IgG Fc antibody (Jackson, West Grove, PA; diluted 1:5,000) was added to each well as a secondary antibody. After incubating for $2 \mathrm{~h}$ at room temperature, 3,3',5,5'-tetramethylbenzidine (TMB) substrate solution (Seracare, Milford, MA) was added to each well, and the color was allowed to develop for 3 min. TMB Stop Solution (Seracare, Milford, MA) was used to stop the reaction. The absorbance of each well was read at $450 \mathrm{~nm}$ on a Gen5 microplate reader with version 2.01 software (Biotek, Winooski, VT).

198

199

\section{Results}

Morphological changes in top, middle, and base leaves after agroinfiltration

201

202

203

204

205

206

207

208

209

210

211

To investigate morphological changes in the plant leaves after agroinfiltration, the leaf morphology of $N$. benthamiana plants agroinfiltrated with Agrobacterium carrying pEAQ-GA733$\mathrm{Fc}(\mathrm{Ag} / \mathrm{pEAQ}-\mathrm{GA} 733-\mathrm{Fc})$ and Ag/pEAQ-GA733-FcK was examined according to days postinfiltration (dpi) and leaf position (top, middle, and base) (Fig. 2). In plants agroinfiltrated with Ag/pEAQ-GA733-Fc, leaf chlorosis was not observed (top, middle, and base leaves) until 3 dpi (Fig. 2A). The base leaves started to show chlorosis at 5 dpi. The middle and top leaves showed chlorosis at 7 dpi (Fig. 2A). Serious necrosis was observed on the base leaves at 8 dpi, while necrosis was observed on the middle and top leaves at 9 and 10 dpi, respectively. In plants agroinfiltrated with Ag/pEAQ-GA733-Fc and Ag/pEAQ-GA733-FcK, chlorosis and necrosis began on the base leaves at 5 and $7 \mathrm{dpi}$, respectively (Fig. 2B). The middle and top leaves both showed chlorosis and necrosis at 7 and 8 dpi, which was $2-3$ days later than the base leaves (Fig. 
212 2B). At $10 \mathrm{dpi}$, all the base leaves agroinfiltrated with both vectors showed necrosis (Fig. 2). After

2137 dpi, regardless of the transgene, all the base leaves showed chlorosis and necrosis with wilting,

214 and the leaf mass decreased rapidly (Fig. 2).

215 PCR confirmation of GA733-Fc and GA733-FcK genes in agroinfiltrated leaves of $N$.

216 benthamiana

217 PCR analysis was conducted to confirm the presence of the genes encoding GA733-Fc and

218 GA733-FcK in the agroinfiltrated leaves at each leaf position (top, middle, and base leaves) (Fig.

219 3). The expected sizes of the amplified GA733-Fc (1,461 bp) and GA733-FcK (1,473 bp) bands

220 were observed regardless of leaf position (Fig. 3). There was no band in the genomic DNA sample

221 from a plant not infiltrated with Agrobacterium (Fig. 3). In plants agroinfiltrated with Ag/pEAQ-

222 GA733-Fc, the top and middle leaves produced PCR bands at all observed dpi (Fig. 3A). All the

223 base leaves at 1, 3, 5, and 7 dpi produced the PCR band. In plants agroinfiltrated with Ag/pEAQ-

224 GA733-FcK, the PCR band trends were similar to those with Ag/pEAQ-GA733-Fc (Fig. 3B). The

225 agroinfiltrated base leaves at 8, 9, and 10 dpi were not included for PCR analysis owing to the lack

226 of sample mass caused by severe chlorosis, necrosis, and wilting.

227 Western blot analysis to determine the expression levels of GA733-Fc and GA733-FcK

228 according to leaf position

229

Western blot analysis was performed to investigate the differences in expression levels of

230

231

232

233

234

235

236

237

238

239

240

241

242

243

244

245 GA733-Fc and GA733-FcK depending on the leaf position (top, middle, and base leaves) and dpi (Fig. 4). To compare the protein expression level, $2 \mu$ of each sample extracted from equal biomasses was loaded in each western blot lane. In GA733-Fc, there were a $65 \mathrm{kDa}$ and $>240 \mathrm{kDa}$ protein bands for the top leaves at 3,5, and $7 \mathrm{dpi}$. At $5 \mathrm{dpi}$, the $65 \mathrm{kDa}$ band density was the strongest among the others in the leaves agroinfiltrated with Ag/pEAQ-GA733-Fc (Fig. 4A left panels). In the middle and base leaves, the $65 \mathrm{kDa}$ band was not detected. However, in the middle leaves at 3-10 dpi, several bands larger than $240 \mathrm{kDa}$ were detected. In plants agroinfiltrated with Ag/pEAQ-GA733-FcK, there were 65 and $240 \mathrm{kDa}$ protein bands for the top leaves at all dpi (Fig. 4A right panel). The middle leaves also showed the $\sim 65$ and $\sim 240 \mathrm{kDa}$ protein bands at 5, 7, 8 , and $9 \mathrm{dpi}$, whereas at 3 and $10 \mathrm{dpi}$, the bands were barely detected (Fig. 4A right middle). In the base leaves, only the $\sim 240 \mathrm{kDa}$ protein band was detected at $8 \mathrm{dpi}$. In general, protein expression was higher for GA733-FcK than for GA733-Fc (Fig. 4B). The top leaf position showed relatively high expression regardless of the vector type (Fig. 4B). There was almost no protein expression in the base leaf position (Fig. 4). From the highest to the lowest, the relative protein density levels were ranked top, middle, and base. In general, the highest band density was observed at 5 dpi in the top leaves (Fig. 4).

Purification of GA733-Fc and GA733-FcK from agroinfected N. benthamiana plant leaves

GA733-Fc and GA733-FcK transiently expressed in N. benthamiana leaves were purified (Fig. 5). The SDS-PAGE analysis showed that the GA733-Fc and GA733-FcK proteins were successfully purified using protein A affinity chromatography (Fig. 5). Monomer protein bands of the expected size $(\sim 65 \mathrm{kDa})$ were detected for both plant-derived GA733-Fc (GA733-Fc $\left.{ }^{\mathrm{P}}\right)$ and GA733-FcK ${ }^{\mathrm{P}}$. The dimeric protein band $(\sim 140 \mathrm{kDa})$ was also observed in the purified GA733$\mathrm{FcK}^{\mathrm{P}}$. The protein band intensity was stronger for GA733-FcK than for GA733-Fc (Fig. 5). Indeed, $1.12 \mathrm{mg}$ of GA733-Fc and $11.82 \mathrm{mg}$ of GA733-FcK were purified from $100 \mathrm{~g}$ of infiltrated $\mathrm{N}$. benthamiana leaves (5 dpi). 
255

256

257

258

259

260

261

262

263

\section{4}

265

266

267

268

269

270

271

272

273

274

275

276

277

278

279

280

281

282

283

284

285

286

287

288

289

290

291

292

293

294

295

296

297

298

ELISA to confirm binding of GA733-Fc ${ }^{\mathrm{P}}$ and $\mathrm{GA} 733-\mathrm{FcK}^{\mathrm{P}}$ proteins to anti-GA733 antibody

ELISA was conducted to compare the binding of $\mathrm{GA} 733-\mathrm{Fc}^{\mathrm{P}}$ and $\mathrm{GA} 733-\mathrm{FcK}^{\mathrm{P}}$ to an antiGA733 antibody. The 96-well plate was coated with anti-EpCAM antibody (anti-GA733) (50 ng/well), and the purified GA733-Fc ${ }^{\mathrm{P}}$ and $\mathrm{GA} 733-\mathrm{FcK}^{\mathrm{P}}$ proteins were applied (Fig. 6B). Antihuman IgG Fc antibody conjugated to horseradish peroxidase (HRP) was used as a secondary antibody to bind the $\mathrm{Fc}$ region of both $\mathrm{GA} 733-\mathrm{Fc}^{\mathrm{P}}$ and $\mathrm{GA} 733-\mathrm{FcK}^{\mathrm{P}}$ (Fig. 6B). The signal from GA733-Fc ${ }^{\mathrm{P}}$ and $\mathrm{GA} 733-\mathrm{FcK}^{\mathrm{P}}$ was higher than that from EpCAM-Fc ${ }^{\mathrm{M}}$. The GA733-FcK ${ }^{\mathrm{P}}$ showed a higher absorbance compared to the GA733-Fc ${ }^{P}$ (Fig. 6).

\section{Discussion}

We demonstrated that the leaf tissue position and days post-infiltration (dpi) affected the transient expression level of recombinant GA733 protein fused to an human immunoglobulin Fc fragment (GA733-Fc) and GA733-Fc fused to an ER retention motif (GA733-FcK) in $N$. benthamiana plant. For transgenic plant expression, a transformation strategy is required to stably integrate the gene of interest into the plant genome. This transgenic approach has some advantages in terms of scaling up production since the transgene is stably inherited through generations and transgenic seeds can be stored for large-scale cultivation (Chen et al. 2013; Daniell et al. 2009; Peyret \& Lomonossoff 2013). However, it has some disadvantages; for instance, generating plant lines requires a long time with low yields of the target protein. On the contrary, plant transient expression system can overcome these drawbacks of time and yield. For transient expression, plant viral or non-viral vectors are utilized to express genes of interest in plants (Chen et al. 2013). Among these strategies, deconstructed viruses that lack the viral coat protein gene allow for production of heterologous proteins in a few days (Alamillo et al. 2006; Chen et al. 2013; Peyret \& Lomonossoff 2013). Elimination of the region encoding the coat protein enhances the transgene capacity of the virus, enabling it to efficiently produce large recombinant proteins (Chen et al. 2013). In addition, the deconstructed virus vector can replicate in plant cells to a higher level than the full-virus system, allowing a larger amount of recombinant protein to be harvested (Chen et al. 2013).

In the present study, we hypothesized that the transient protein expression levels of GA733Fc and GA733-FcK varied depending on the leaf position (top, middle, and base) and transfection periods. Thus, the ultimate goal of this study was to optimize the leaf position for agroinfiltration and leaf harvesting time to enhance production of GA733-Fc and GA733-FcK using the plant transient expression system. First, the morphological changes in the leaves (top, middle, and base) at the dpi were investigated. According to the data, 5-7 dpi was the best time for leaf harvesting and purification of recombinant protein since the leaves during these days showed the least damage (i.e., chlorosis, necrosis, and wilting), and the area of the leaves did not reduce when compared to those on the other later days. The loss of chloroplasts results in loss of energy-generating capacity and reduced protein production capacity (Munné-Bosch \& Alegre 2002). Thus, the chlorosis, necrosis, and wilting of leaves induced by Agrobacterium infiltration might affect production of the recombinant protein.

To confirm GA733-Fc and GA733-FcK gene insertion, PCR analysis was conducted. The expected sizes of the amplified PCR bands were observed (GA733-Fc: 1,461 bp, GA733-FcK: 1,473 bp). However, the base leaves of both GA733-Fc and GA733-FcK plants at 8, 9, and 10 dpi were not included in the analysis since the sample mass was insufficient for PCR analysis owing 
299 to chlorosis, necrosis, and wilting. All the tested leaf samples produced the expected PCR products, 300 indicating that the infiltration properly delivered both the GA733-Fc and GA733-FcK gene 301 expression cassettes. However, the presence of the transgene in the leaf does not necessarily mean 302 expression of the GA733-Fc and GA733-FcK proteins. Thus, western blotting was carried out to 303 confirm their protein expression. The band of mammalian-derived GA733-Fc was slightly 304 different from plant-derived GA733-Fc and GA733-FcK. It is speculated that the discrepancy is 305 due to difference between mammalian and plant glycosylation, which affects molecular weight, 306 altering protein migration on a western blot (Unal et al. 2008). Accordingly, the protein expression 307 of GA733-Fc and GA733-FcK increased until 5 dpi in the top and middle leaves and then 308 decreased, while the protein expression level in the base leaves was relatively low. In the leaves 309 infiltrated with Agrobacterium carrying the GA733-Fc expression vector, the GA733-Fc protein $310(\sim 65 \mathrm{kDa})$ was detected in the upper leaves only at 3, 4, and 5 dpi. The fluctuation of protein 311 expression of GA733-FcK is due to the extreme transient expression of foreign proteins localized 312 in ER during 5 7 dpi resulting in temporary plant cell stress such as ER stress (Howel 2013) or 313 temporary protease expression and activation (Mandal et al. 2016). In addition, the western blot 314 analysis showed that the protein expression level was higher in the leaves infiltrated with 315 Agrobacterium carrying the GA733-FcK expression vector than in the leaves infiltrated with the 316 GA733-Fc expression vector. These results indicate that the KDEL ER retention signal affected 317 the production level of the GA733-Fc fusion protein in the transient plant expression system. In 318 previous studies, KDEL tagging to recombinant antibodies and antigens localized and accumulated 319 them in the ER, eventually increasing their production levels in transgenic plants (Kang et al. 2016; 320 Lee et al. 2013; Lee et al. 2020; Park et al. 2020; So et al. 2013; Song et al. 2018). Indeed, $N$ glycosylation analysis showed that GA733-FcK had mainly oligo-mannose compared to GA733Fc, indicating the KDEL induced ER retention of GA733-FcK (Fig. S1).

Both GA733-Fc and GA733-FcK expression levels in the leaves decreased in the order of top, middle, and base. The bottom leaves become the main leaves first after germination. These leaves are older with fewer chloroplasts, resulting in lower energy and protein production than the middle and top leaves, which are younger (Munné-Bosch \& Alegre 2002). Such low recombinant protein expression is probably related to the low concentration of total soluble protein in the old leaves (Halfhill et al. 2003).

For purification of both GA733-Fc and GA733-FcK, protein A affinity chromatography was utilized since the Fc domain was fused to the GA733 protein (Lee et al. 2020; Park et al. 2015; Park et al. 2020; Shin et al. 2019). SDS-PAGE showed that the purified GA733-FcK had stronger band intensity than the purified GA733-Fc. The concentration of the purified GA733-Fc and GA733-FcK proteins was 0.112 and $1.82 \mathrm{mg} / \mathrm{ml}$, respectively, representing 16.25 times higher production of GA733-FcK than GA733-Fc. The enhanced protein production of GA733-FcK in the current study is consistent with that in a previous study, where the transient expression of ERtargeted human interleukin- 6 represented $7 \%$ of the total soluble protein (TSP) in $N$. benthamiana (Munné-Bosch \& Alegre 2002). We speculate that the ER-retained GA733-Fc protein molecules interacted with ER-resident chaperones to promote correct folding (Benchabane et al. 2008) and were less exposed to proteases in the ER (Vitale \& Pedrazzini 2005) in transient plant system.

In this present study, we confirmed that the protein expression varied with the dpi. After agroinfiltration of the GA733-FcK vector, the top leaves began to express the protein at 3 dpi, with strong expression from 5 to $9 \mathrm{dpi}$, except for $7 \mathrm{dpi}$. For the middle leaves, the highest expression was observed at $5 \mathrm{dpi}$. These results suggest that the best leaf harvesting time for purification of GA733-FcK proteins is $5 \mathrm{dpi}$ and that GA733-Fc can be transiently expressed using the CPMV

Peer) reviewing PDF | (2020:08:51767:1:1:NEW 22 Dec 2020) 
345

346

347

348

349

350

351

352

353

354

355

356

357

358

359

360

361

362

363

364

365

366

367

368

369

370

371

372

373

374

375

376

377

378

379

380

381

382

383

384

385

386

virus-based vector with an optimized transfection period and leaf position. According to the current results, this harvesting time is applicable for top leaves infiltrated with Agrobacterium carrying the GA733-Fc vector. Previous studies also showed high expression of recombinant proteins at 4-6 dpi (Norkunas et al. 2018). The leaf tissue position and dpi affect both GA733-Fc and GA733-FcK recombinant protein expression. Thus, to optimize protein expression, the top leaves should be infiltrated and then harvested from 5 to 9 dpi, and not before 3 dpi and after 9 dpi.

Sandwich ELISA was performed to detect the binding of each purified GA733-Fc and GA733FcK protein to an anti-GA733 antibody. The resulting absorbance of GA733-FcK was stronger than that of GA733-Fc. These results suggest that the antibody is able to bind GA733-FcK better than GA733-Fc, and they are similar to our previous studies where recombinant proteins tagged with KDEL showed better interactions with antibodies regardless of the specific antigen or antibody (Kang et al. 2016; Lee et al. 2013; So et al. 2013; Song et al. 2018). We speculate that ER-retained recombinant proteins are more likely to be correctly folded or favorably glycosylated for better binding activity than cytosolic or apoplastic proteins. In the surface plasmon resonance (SPR) analysis (Fig. S2), in addition, GA733-FcK had higher affinity for Fc $\gamma R$ I (CD64) than GA733-Fc, indicating that the Fc domain of GA733-FcK interacts better with Fc $\gamma R$ I (CD64) than that of GA733-Fc.

\section{Conclusions}

In this study, we determined the optimal leaf position and dpi for the transient expression of GA733-Fc and GA733-FcK. In addition, we confirmed that KDEL tagging of GA733-Fc enhanced its production in the transient plant expression system. Taken together, the Fc fusion protein GA733-Fc can be expressed using a CPMV virus-based vector in transient plant system. Plants are in the spotlight as an excellent therapeutic protein production platform. We have expressed and purified colorectal cancer vaccine recombinant protein GA733-Fc and GA733-Fc with KDEL (GA733-FcK) by transient expression system using pEAQ-HT vector with reduced labor than transgenic expression system. By using our currently described optimized GA733-Fc and GA733FcK transient expression condition, it is expected that mass production of GA733-Fc and GA733FcK would be realized in the future.

\section{Acknowledgements}

We thank Professor George Lomonossoff (John Innes Centre, Norwich, United Kingdom) for providing the plant viral pEAQ- $H T$ vector. This study was conducted in Department of Medicine, Therapeutic Protein Engineering Lab, College of Medicine, Chung-Ang University.

\section{References}

Alamillo JM, Monger W, Sola I, García B, Perrin Y, Bestagno M, Burrone OR, Sabella P, Plana-Durán J, and Enjuanes L Biotechnology journal. 2006. Use of virus vectors for the expression in plants of active full-length and single chain anti-coronavirus antibodies. 1:1103-1111.

PeerJ reviewing PDF | (2020:08:51767:1:1:NEW 22 Dec 2020) 
387 Benchabane M, Goulet C, Rivard D, Faye L, Gomord V, and Michaud D Plant biotechnology

388

389

390

391

392

393

394

395

396

397

398

399

400

401

402

403

404

405

406

407

408

409

410

411

412

413

414

415

416

417

418

419

420

421

422

423

424

425

426

427

428

429

430

431 journal. 2008. Preventing unintended proteolysis in plant protein biofactories. 6:633-648.

Brodzik R, Spitsin S, Golovkin M, Bandurska K, Portocarrero C, Okulicz M, Steplewski Z, and Koprowski H Cancer Immunol Immunother. 2008. Plant-derived EpCAM antigen induces protective anti-cancer response. 57:317-323.

Chen Q, Lai H, Hurtado J, Stahnke J, Leuzinger K, Dent M NIH Public Access. 2013. Agroinfiltration as an effective and scalable strategy of gene delivery for production of pharmaceutical proteins. 1 .

Czajkowsky DM, Hu J, Shao Z, and Pleass RJ EMBO Molecular Medicine. 2012. Fc-fusion proteins: new developments and future perspectives. 4:1015-1028.

Daniell H, Singh ND, Mason H, and Streatfield SJ Trends in Plant Science. 2009. Plant-made vaccine antigens and biopharmaceuticals. 14:669-679.

Eun-Wha Son, Sang-Whan In, and Suhkneung Pyo Immune Network. 2005. Cancer Vaccines. 5:55-67.

Ersin Selcuk Unal, Roongbao Zhao, Andong Qui, and I. David Goldman Biochim Biophys Acta Biomembr. 2008. N-linked glycosylation and its impact on the electtrophoretic mobility and function of the human proton-coupled folate transporter (HsPCFT). 1407-1414.

Eyvazi S, Farajnia S, Dastmalchi S, Kanipour F, Zarredar H, and Bandehpour M Current Cancer Drug Targets. 2018. Antibody based EpCAM targeted therapy of cancer, review and update. $18: 857-868$.

Halfhill M, Millwood R, Weissinger A, Warwick S, Stewart C Theoretical and Applied Genetics. 2003. Additive transgene expression and genetic introgression in multiple greenfluorescent protein transgenic crop $\times$ weed hybrid generations. 107:1533-1540.

Joh LD, McDonald KA, and VanderGheynst JS Biotechnology Progress. 2006. Evaluating extraction and storage of a recombinant protein produced in agroinfiltrated lettuce. 22:723-730.

Kang Y, Shin YK, Park S-W, Ko K Horticulture, Environment, and Biotechnology. 2016. Effect of nitrogen deficiency on recombinant protein production and dimerization and growth in transgenic plants. 57:299-307.

Kim D-S, Song I, Ko K Horticulture, Environment, and Biotechnology. 2018. Low risk of pollenmediated gene flow in transgenic plants under greenhouse conditions. 59:723-728.

Lee J-H, Park D-Y, Lee K-J, Kim Y-K, So Y-K, Ryu J-S, Oh S-H, Han Y-S, Ko K, and Choo Y$\mathrm{K}$ Plos One. 2013. Intracellular reprogramming of expression, glycosylation, and function of a plant-derived antiviral therapeutic monoclonal antibody. 8.

Lee JH, Ko K Biomolecules \& Therapeutics, and therapeutics. 2017. Production of recombinant anti-cancer vaccines in plants. 25:345.

Lee JH, Park SR, Phoolcharoen W, and Ko K Plant Biotechnology Reports. 2020. Expression, function, and glycosylation of anti-colorectal cancer large single-chain antibody (LSC) in plant.1-9.

Lim C-Y, Lee KJ, Oh D-B, and Ko K Frontiers in Plant Science. 2015. Effect of the developmental stage and tissue position on the expression and glycosylation of recombinant glycoprotein GA733-FcK in transgenic plants. 5:778.

Loh H-S, and Wayah S Asian Journal of Biotechnology. 2014. Optimizations of laboratoryscale vacuum-assisted agroinfiltration for delivery of a transgene in Nicotiana benthamiana. $6: 1-14$. 
432 Lu Z, Lee K-J, Shao Y, Lee J-H, So Y, Choo Y-K, Oh D-B, Hwang K-A, Oh SH, and Han YS

433

434

435

436

437

438

439

440

441

442

443

444

445

446

447

448

449

450

451

452

453

454

455

456

457

458

459

460

461

462

463

464

465

466

467

468

469

470

471

472

473

474

475

476

BioMed Research International. 2012. Expression of GA733-Fc fusion protein as a vaccine candidate for colorectal cancer in transgenic plants. 2012.

Ma T, Li ZY, and Wang S Frontiers in Plant Science. 2019. Production of Bioactive Recombinant rPA (Reteplase) by Virus-based Transient Expression System in Nicotiana benthamiana. 10:1225.

Menassa R, Ahmad A, and Joensuu JJ Molecular farming in plants: recent advances and future prospects: Springer. 2012. Transient expression using agroinfiltration and its applications in molecular farming. 183-198.

Munné-Bosch S, and Alegre L Planta. 2002. Plant aging increases oxidative stress in chloroplasts. 214:608-615.

Nausch H, Mikschofsky H, Koslowski R, Meyer U, Broer I, and Huckauf J Plos One. 2012. Highlevel transient expression of ER-targeted human interleukin 6 in Nicotiana benthamiana. 7.

Norkunas K, Harding R, Dale J, and Dugdale B Plant Methods. 2018. Improving agroinfiltrationbased transient gene expression in Nicotiana benthamiana. 14:1-14.

Park SR, Lim C-Y, Kim D-S, and Ko K Frontiers in Plant Science. 2015. Optimization of ammonium sulfate concentration for purification of colorectal cancer vaccine candidate recombinant protein GA733-FcK isolated from plants. 6:1040.

Park SR, Lee J-H, Kim K, Kim TM, Lee SH, Choo Y-K, Kim KS, and Ko K International Journal of Molecular Sciences. 2020. Expression and In Vitro Function of Anti-Breast Cancer Llama-Based Single Domain Antibody VHH Expressed in Tobacco Plants. 21:1354.

Peyret H, and Lomonossoff GP Plant Molecular Biology. 2013. The pEAQ vector series: the easy and quick way to produce recombinant proteins in plants. 83:51-58.

Rigano MM, Walmsley AM Immunology and Cell Biology. 2005. Expression systems and developments in plant-made vaccines. 83:271-277.

Sainsbury F, Thuenemann EC, and Lomonossoff GP Plant Biotechnology Journal. 2009. pEAQ: versatile expression vectors for easy and quick transient expression of heterologous proteins in plants. 7:682-693.

Se-Ra Park. 2019. Expression and functional analysis of immunotherapeutic recombinant proteins in heterologous host systems. The Graduate School Chung-Ang University.

Shah KH, Almaghrabi B, and Bohlmann H Plant Molecular Biology Reporter. 2013. Comparison of expression vectors for transient expression of recombinant proteins in plants. 31:15291538.

Shin C, Kang Y, Kim H-S, Shin YK, Ko K Animal Cells and Systems. 2019. Immune response of heterologous recombinant antigenic protein of viral hemorrhagic septicemia virus (VHSV) in mice. 23:97-105.

So Y, Lee K-J, Kim D-S, Lee J-H, Oh D-B, Hwang K-A, Ko K, Choo Y-K, Ko K Plant Cell, Tissue and Organ Culture. 2013. Glycomodification and characterization of anti-colorectal cancer immunotherapeutic monoclonal antibodies in transgenic tobacco. 113:41-49.

Song I, Kang Y, Lee YK, Myung S-C, and Ko K Plos One. 2018. Endoplasmic reticulum retention motif fused to recombinant anti-cancer monoclonal antibody (mAb) CO17-1 A affects mAb expression and plant stress response. 13.

Stephen H. Howell Annual Review of Plant Biology. 2016. Endoplasmic reticulum stress responses in plants. 64:477-499

Peer) reviewing PDF | (2020:08:51767:1:1:NEW 22 Dec 2020) 
477

478

479

480

481

482

483

484

485

486

487

488

489

490

491

492

493

494

495

496

497

498

499

500

501

502

503

504

505

506

507

508

509

510

511

512

513

514

515

516

517

518

Vitale A, and Pedrazzini E Molecular Interventions. 2005. Recombinant pharmaceuticals from plants: the plant endomembrane system as bioreactor. 5:216.

Wong-Arce A, González-Ortega O, and Rosales-Mendoza S Trends in Biotechnology. 2017. Plantmade vaccines in the fight against cancer. 35:241-256.

Wydro M, Kozubek E, and Lehmann P The Journal of the Polish Biochemical Society and of the Polish Academy of Sciences. 2006. Optimization of transient Agrobacterium-mediated gene expression system in leaves of Nicotiana benthamiana. 53:289-298.

Yamamoto T, Hoshikawa K, Ezura K, Okazawa R, Fujita S, Takaoka M, Mason HS, Ezura H, and Miura K Scientific Reports. 2018. Improvement of the transient expression system for production of recombinant proteins in plants. 8:1-10.

\section{Legends of figure}

Figure 1. Schematic diagram of transient expression vectors for GA733-Fc (A) and GA733-FcK (B) proteins. GA733-Fc and GA733-FcK gene expression cassettes were introduced into the pEAQ-HT plant transient expression vector. 35SP, Cauliflower mosaic virus 35S promoter; 5UTR, Cowpea mosaic virus RNA-2 5'-UTR; 3UTR, Cowpea mosaic virus RNA-2 3'-UTR; P, suppressor of gene silencing (P19); N II, kanamycin resistance gene (NPT II); K, endoplasmic reticulum retention signal (KDEL); NOST, nopaline synthase gene terminator; 35ST, Cauliflower mosaic virus $35 \mathrm{~S}$ terminator. Expected protein structure of the recombinant fusion proteins GA733-Fc and GA733-FcK: green oval-shaped bar, GA733; connected yellow bar, Fc domain; blue star-shaped region, KDEL. Expected glycan structure: the symbols of the glycan structures are as follows: $N$ acetylglucosamine, gray square; mannose, white circle; overlapped white diamonds, $\alpha(1,3)$ fucose; white triangle, $\beta(1,2)$-xylose.

Figure 2. Physical appearance of $N$. benthamiana leaves (top, middle, and base leaves) infiltrated with Agrobacterial inoculum carrying pEAQ-GA733-Fc (Ag/pEAQ-GA733-Fc) (A) and pEAQGA733-FcK (Ag/pEAQ-GA733-FcK) (B) for 1, 3, 5, 7, 8, 9, and 10 days post-infection (dpi). $N$. benthamiana plants were grown for $4-5$ weeks in a greenhouse at $24^{\circ} \mathrm{C}$ and $30 \%$ humidity, with $16 \mathrm{~h}$ light and $8 \mathrm{~h}$ dark. Six-week-old plants with similar number of leaves were used in this study.

Figure 3. PCR analysis of genomic DNA from plant leaves (top, middle, and base) at days post infiltration (dpi) with Ag/pEAQ-GA733-Fc and Ag/pEAQ-GA733-FcK. Genomic DNA was extracted from plant leaves expressing GA733-Fc (A) and GA733-FcK (B) according to days post-infection (dpi) and leaf position (top, middle, and base leaves), and the targeted genes were amplified and separated on a 1\% agarose gel by electrophoresis. The $8-10$ dpi samples of base leaves for both GA733-Fc and GA733-FcK were insufficient for PCR. +: positive control (GA733-Fc and GA733-FcK plasmid DNA extracted from Escherichia coli), -: negative control (non-infiltrated plant): 1-10 days post-infiltration samples.

Figure 4. Comparison of expression levels at various times $[1,3,5,7,8$, 9, and 10 days postinfiltration (dpi)] and leaf position (top, middle, and base) by western blot analysis (A), and relative band intensity of GA733-Fc and GA733-FcK protein bands $(\sim 65 \mathrm{kDa})$ with respect to dpi (B). Western blot analysis was performed to identify GA733-Fc and GA733-FcK protein expression levels in accordance with various dpi using agroinfiltrated leaves of $N$. benthamiana. GA733-Fc and GA733-FcK were detected using murine anti-GA733 IgGs and 
519 anti-murine IgG IgGs conjugated to horseradish peroxidase. +, positive control [mammalian520 derived GA733-Fc (GA733-Fc $\left.{ }^{\mathrm{M}}\right), 70 \mathrm{ng}$ ]; -, negative control (non-infiltrated plant); 1-10 dpi, 521 samples. (B) The graph shows relative band densities of GA733-Fc (W/O) and GA733-FcK 522 (W) proteins. The crude leaf extract was applied for western blot.

523 Figure 5. SDS-PAGE of purified GA733-Fc $\mathrm{P}^{\mathrm{P}}(\mathrm{W} / \mathrm{O})$ and $\mathrm{GA} 733-\mathrm{FcK}^{\mathrm{P}}(\mathrm{W})$ from infiltrated 524 leaves of $N$. benthamiana plants. The purified $\mathrm{GA} 733-\mathrm{Fc}^{\mathrm{P}}$ and $\mathrm{GA} 733-\mathrm{FcK}^{\mathrm{P}}$ were analyzed 525 by $10 \%$ SDS-PAGE. + , GA733-Fc ${ }^{\mathrm{M}}(1 \mu \mathrm{g})$ as a positive control.

526 Figure 6. SDS-PAGE to confirm equal amounts of purified GA733-Fc ${ }^{\mathrm{P}}$ and $\mathrm{GA} 733-\mathrm{FcK}^{\mathrm{P}}$ proteins 527 and sandwich ELISA to assess binding activity of anti-GA733 antibody to both GA733-Fc ${ }^{\mathrm{P}}$ and 528 GA733-FcK $^{\mathrm{P}}$. Loading of the same quantity of GA733-Fc ${ }^{\mathrm{P}}$ and $\mathrm{GA} 733-\mathrm{FcK}^{\mathrm{P}}$ proteins in ELISA 529 was confirmed by SDS-PAGE (A). Schematic representation of the sandwich ELISA (B). Results 530 of ELISA for GA733-Fc ${ }^{\mathrm{M}}, \mathrm{GA} 733-\mathrm{Fc}^{\mathrm{P}}$, and $\mathrm{GA} 733-\mathrm{FcK}^{\mathrm{P}}(\mathrm{C})$. 


\section{Figure 1}

Schematic diagram of transient expression vectors for GA733-Fc (A) and GA733-FcK (B) proteins.

GA733-Fc and GA733-FcK gene expression cassettes were introduced into the pEAQ-HT plant transient expression vector. 35SP, Cauliflower mosaic virus 35S promoter; 5UTR, Cowpea mosaic virus RNA-2 5'-UTR; 3UTR, Cowpea mosaic virus RNA-2 3'-UTR; P, suppressor of gene silencing (P19); NII, kanamycin resistance gene (NPTII); K, endoplasmic reticulum retention signal (KDEL); NOST, nopaline synthase gene terminator; 35ST, Cauliflower mosaic virus 35S terminator. Expected protein structure of the recombinant fusion proteins GA733-Fc and GA733-FcK: green oval-shaped bar, GA733; connected yellow bar, Fc domain; blue starshaped region, KDEL. Expected glycan structure: the symbols of the glycan structures are as follows: $N$-acetylglucosamine, gray square; mannose, white circle; overlapped white diamonds, $\alpha(1,3)$-fucose; white triangle, $\beta(1,2)$-xylose.

A

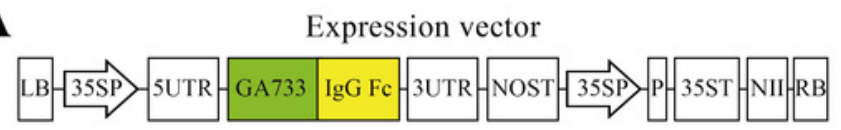

Expected protein structure

Expected $N$-glycan structure

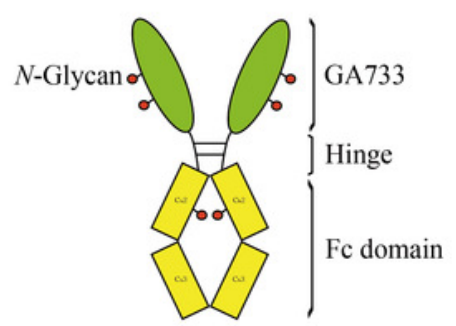

pEAQ-GA733-Fc
B

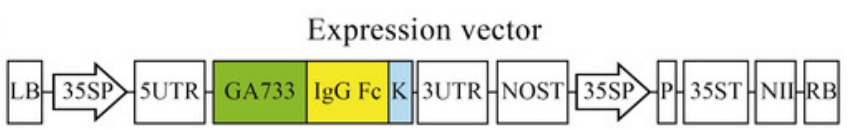

Expected protein structure

Expected $\mathrm{N}$-glycan structure
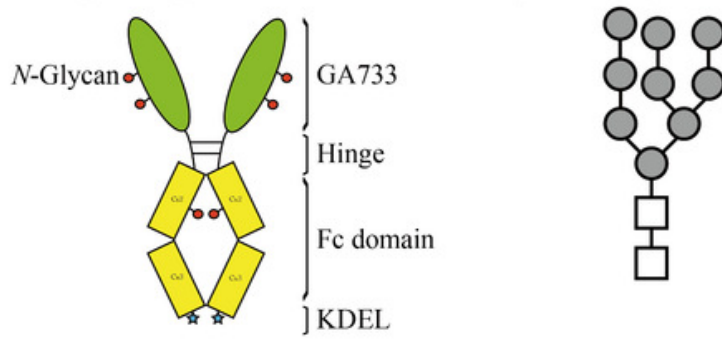

pEAQ-GA733-FcK 


\section{Figure 2}

Physical appearance of $N$. benthamiana leaves (top, middle, and base leaves) infiltrated with Agrobacterial inoculum carrying pEAQ-GA733-Fc (Ag/pEAQ-GA733-Fc) (A) and pEAQ-GA733-FcK (Ag/pEAQ-GA733-FcK) (B) for 1, 3, 5, 7, 8, 9, and 10 days post-infec

$N$. benthamiana plants were grown for $4-5$ weeks in a greenhouse at $24^{\circ} \mathrm{C}$ and $30 \%$ humidity, with $16 \mathrm{~h}$ light and $8 \mathrm{~h}$ dark. Six-week-old plants with similar number of leaves were used in this study.
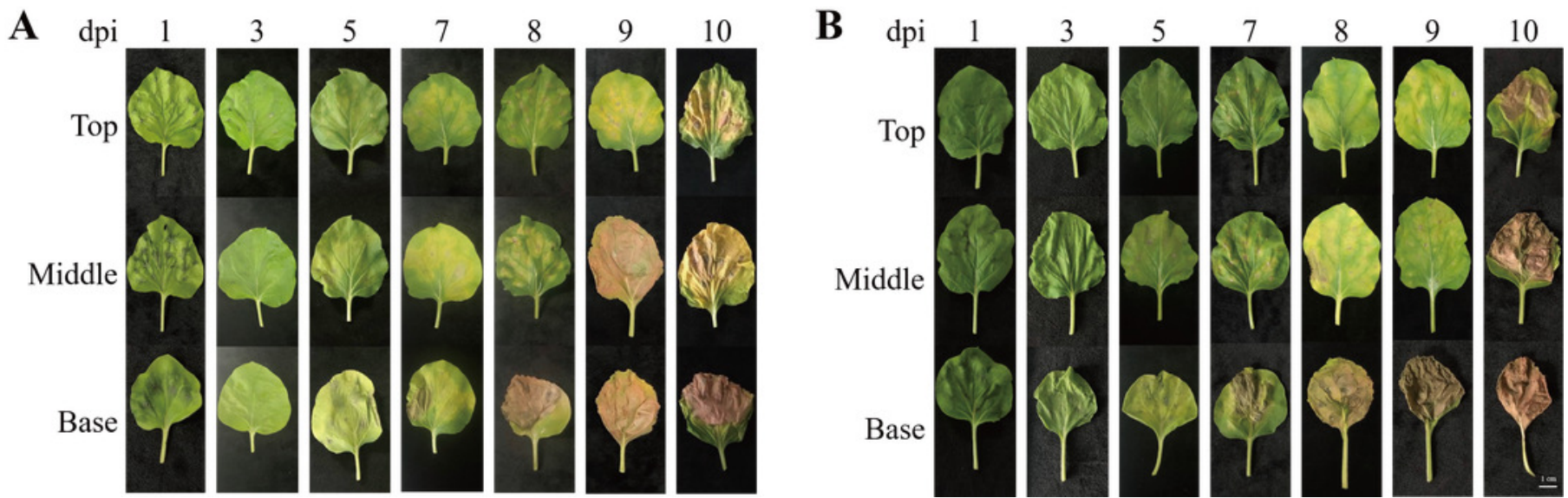


\section{Figure 3}

PCR analysis of genomic DNA from plant leaves (top, middle, and base) at days post infiltration (dpi) with Ag/pEAQ-GA733-Fc and Ag/pEAQ-GA733-FcK.

Genomic DNA was extracted from plant leaves expressing GA733-FC (A) and GA733-FcK (B) according to days post-infection (dpi) and leaf position (top, middle, and base leaves), and the targeted genes were amplified and separated on a 1\% agarose gel by electrophoresis. The 8-10 dpi samples of base leaves for both GA733-Fc and GA733-FcK were insufficient for PCR. +: positive control (GA733-Fc and GA733-FcK plasmid DNA extracted from Escherichia coli), -: negative control (non-infiltrated plant): 1-10 days post-infiltration samples.

$\mathbf{A}$

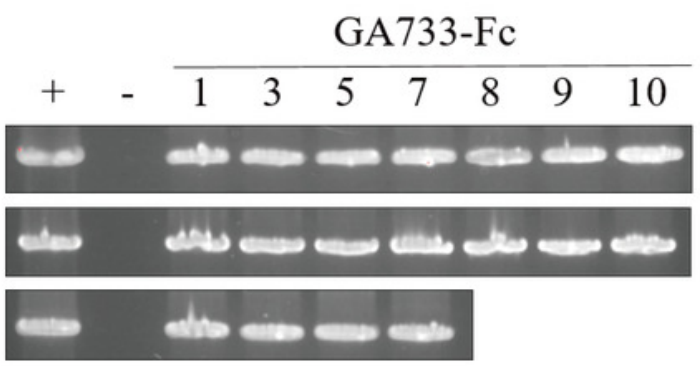

B

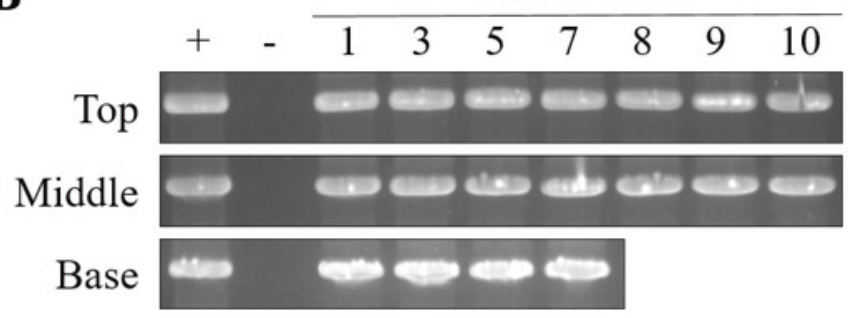




\section{Figure 4}

Comparison of expression levels at various times $[1,3,5,7,8,9$, and 10 days postinfiltration (dpi)] and leaf position (top, middle, and base) by western blot analysis (A), and relative band intensity of GA733-Fc and GA733-FcK protein bands ( $65 \mathrm{kDa}) \mathrm{w}$

Western blot analysis was performed to identify GA733-Fc and GA733-FcK protein expression levels in accordance with various dpi using agroinfiltrated leaves of $N$. benthamiana. GA733Fc and GA733-FcK were detected using murine anti-GA733 IgGs and anti-murine IgG IgGs conjugated to horseradish peroxidase. +, positive control [mammalian-derived GA733-Fc (GA733-FC ${ }^{\mathrm{M}}$ ), $\left.70 \mathrm{ng}\right] ;$-, negative control (non-infiltrated plant); 1-10 dpi, samples. (B) The graph shows relative band densities of GA733-Fc (W/O) and GA733-FcK (W) proteins. The crude leaf extract was applied for western blot.

A

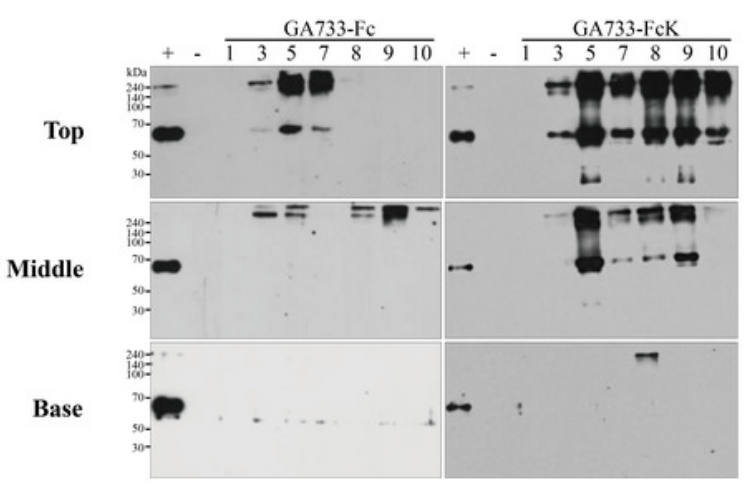

B

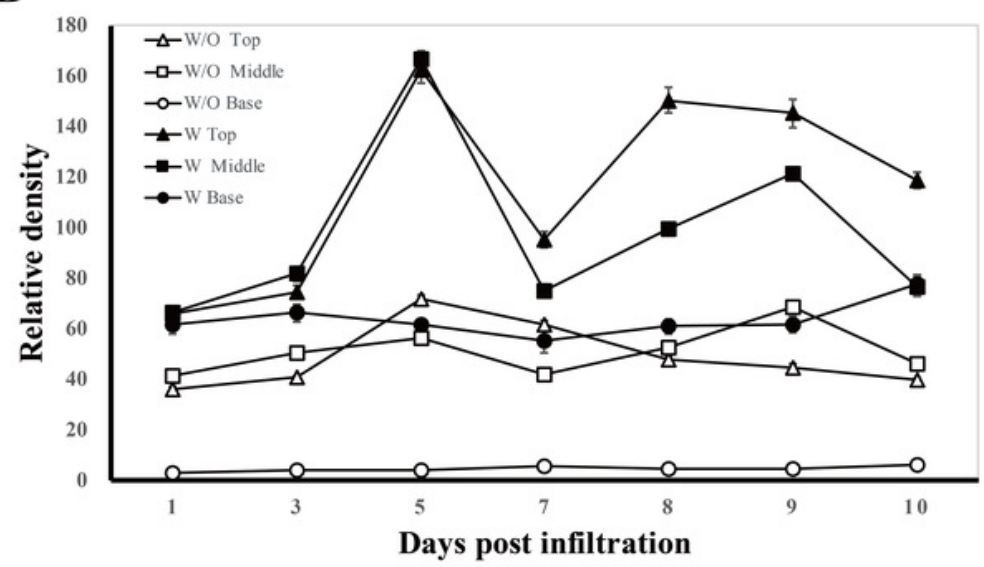


Figure 5

SDS-PAGE of purified GA733-FC ${ }^{P}(W / O)$ and GA733-FCK $K^{P}(W)$ from infiltrated leaves of $N$. benthamiana plants.

The purified GA733-Fc ${ }^{p}$ and GA733-FcK ${ }^{p}$ were analyzed by $10 \%$ SDS-PAGE. + , GA733-Fc ${ }^{M}(1$ $\mu \mathrm{g})$ as a positive control.

$\mathrm{kDa}$
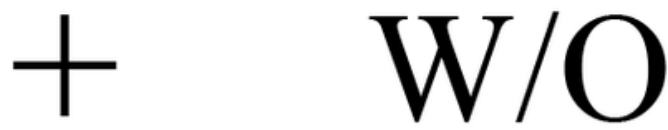

W

140

100

70

50 
Figure 6

SDS-PAGE to confirm equal amounts of purified GA733-FC ${ }^{p}$ and GA733-FCK ${ }^{p}$ proteins and sandwich ELISA to assess binding activity of anti-GA733 antibody to both GA733-Fc ${ }^{p}$ and GA733-FcK.

Loading of the same quantity of GA733-Fc ${ }^{p}$ and GA733-FCK $K^{p}$ proteins in ELISA was confirmed by SDS-PAGE (A). Schematic representation of the sandwich ELISA (B). Results of ELISA for GA733-FC ${ }^{M}$, GA733-FC ${ }^{p}$, and GA733-FCK $(C)$. 
(A)

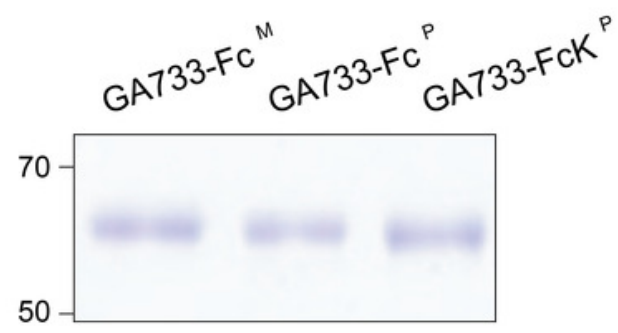

(C)

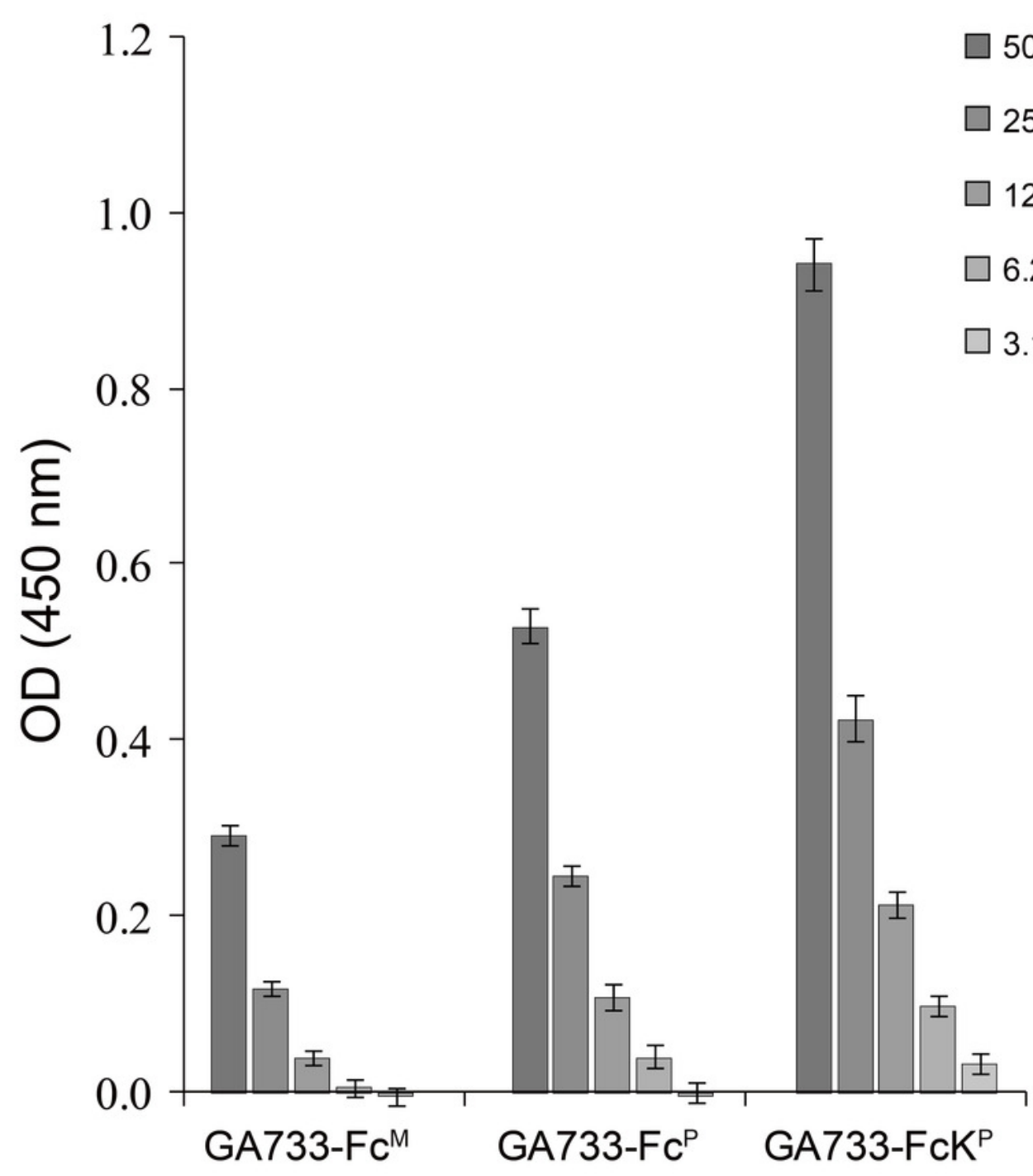

Goat anti-human IgG Fc Antibody conjugated to $\operatorname{HRP}(1: 5,000)$

GA733-Fc ${ }^{M}$ or GA733-Fc ${ }^{P}$ or GA733-FcK ${ }^{P}$

Anti-EpCAM antobody (50 ng/well)

$50 \mathrm{ng}$

$25 \mathrm{ng}$

$12.5 \mathrm{ng}$

$6.25 \mathrm{ng}$

$3.125 \mathrm{ng}$ 